\title{
Influence de l'âge sur les durées de réanimation des arrêts cardiaques préhospitaliers
}

\author{
Impact of Age on Out of Hospital Cardiac Arrest Resuscitation Duration
}

\author{
C. Adt $\cdot$ J. Salignon $\cdot$ Y. Freund $\cdot$ E. Espinasse $\cdot$ P. Ray $\cdot$ A. Avondo \\ Reçu le 12 avril 2018; accepté le 13 juillet 2018 \\ (C) SFMU et Lavoisier SAS 2018
}

Résumé Introduction : L'objectif de notre étude est de déterminer si l'âge des patients en arrêt cardiaque (AC) a une influence sur les durées de réanimation cardiopulmonaire (RCP) par les équipes préhospitalières.

Patients et méthodes : Nous avons réalisé une étude monocentrique, prospective, à partir des données de notre centre hospitalier universitaire, issues du Registre électronique des arrêts cardiaques. Ont été inclus tous les patients ayant présenté un $\mathrm{AC}$, hormis ceux retrouvés en état de rigidité cadavérique ou qui avaient préalablement exprimé des directives anticipées sur leur fin de vie. Les patients ont été séparés en deux groupes selon leur âge : les moins de 75 ans et ceux de 75 ans et plus. Le critère de jugement principal était la durée de RCP spécialisée décidée par le médecin de l'équipe préhospitalière.

Résultats : Du $1^{\text {er }}$ janvier au 31 décembre 2015, sur 253 patients victimes d'AC, 188 (74\% d'hommes, $78 \%$

C. Adt $\cdot$ J. Salignon $\cdot$ E. Espinasse $\cdot$ A. Avondo

Centre régional universitaire d'urgence,

CHU François-Mitterrand,

14, rue Paul-Gaffarel, F-21000 Dijon, France

C. Adt

Centre hospitalier Joigny, 3, quai de l'Hôpital,

F-89300 Joigny, France

\section{Y. Freund}

Service d'accueil des urgences,

Hôpital Pitié-Salpêtrière,

Assistance publique-Hôpitaux de Paris (APHP),

47-83, boulevard de l'Hôpital,

F-75013 Paris, France

Faculté de médecine,

Sorbonne Université,

F-75013 Paris, France

P. Ray $(\bowtie)$

Centre régional universitaire des urgences,

CHU François-Mitterrand, Université de Bourgogne,

14, rue Paul-Gaffarel, F-21000 Dijon, France

e-mail : patrick.ray@chu-dijon.fr d'asystolie) ont bénéficié d'une RCP par une équipe du Service mobile d'urgence et de réanimation. Il y a eu $39 \%$ de récupération d'une activité cardiaque spontanée (RACS). Seuls $31 \%$ des patients étaient admis vivants à l'hôpital, ils étaient $6 \%$ à j30. La durée de RCP était plus importante pour les patients de moins de 75 ans $(29 \pm 15$ vs $23 \pm$ 19 minutes ; $p<0,01)$. Mais pour les patients ayant une RACS, la durée de RCP était identique entre les deux groupes $(16 \pm 10$ vs $14 \pm 9$ minutes ; $p=0,34)$. La survie des patients de 75 ans et plus était de 10 vs $22 \%$ pour les moins de 75 ans $(p=0,35)$.

Conclusion : Notre étude suggère que l'âge des patients influence négativement les durées de réanimation des équipes préhospitalières.

Mots clés Arrêt cardiaque · Âge · Durée de réanimation Pronostic neurologique

Abstract Introduction: Our study's aim is to evaluate if the age of patients who have an out-of-hospital cardiac arrest (OHCA) has an influence on cardiopulmonary resuscitation (CPR) duration carried out by professional teams.

Patients and methods: We performed a prospective, monocentric study, using data from our University Hospital Center, from the National Out-of-Hospital Cardiac Arrest Electronic Register (OHCAR). Patients who had an OHCA were included, except those found in cadaveric rigidity or who had previously expressed advance end-of-life directives. They were divided into two groups according to their age: under 75 years, and 75 years and more. The primary endpoint was the resuscitation duration decided by the prehospital team's physician.

Results: From January 1, 2015 to December 31, 2015, 188/253 (74\% male) OHCA patients received CPR by prehospital medical team. There has been $39 \%$ of return of spontaneous circulation (ROSC). Only $31 \%$ were admitted alive to hospital, $6 \%$ were alive at day 30 . The RCP duration was statistically longer for patients under 75 years old $(29 \pm 15$ 
vs $23 \pm 19$ min; $P<0.01)$. However, for patients with spontaneous cardiac activity return, CPR duration was the same $(16 \pm 10$ vs $14 \pm 9$ min respectively; $P=0.34)$. Survival of patients 75 years and older was 10 vs $22 \%(P=0.35)$.

Conclusion: Our study suggests that as the patient's age increases, the medical teams reduce the duration of resuscitation.

Keywords Cardiac arrest $\cdot$ Age $\cdot$ Prognostic $\cdot$ Duration of resuscitation

\section{Introduction}

Plus de 50000 personnes sont victimes d'un arrêt cardiaque (AC) chaque année en France. Leur pronostic est conditionné par la mise en place de la chaîne de survie comprenant la reconnaissance rapide de l'AC, l'appel des secours, la réalisation précoce d'une réanimation cardiopulmonaire ( $\mathrm{RCP})$, l'obtention d'un défibrillateur et une défibrillation rapide si nécessaire avant une prise en charge spécialisée par des secours professionnels. Au sein des Samu-Centre 15 (Service d'aide médicale urgente), les assistants de régulation médicale (ARM) et les médecins régulateurs du Centre de réception et de régulation des appels (CRRA) doivent reconnaître rapidement les signes d'AC afin d'envoyer les secours adaptés et de débuter au plus vite une réanimation conduite par téléphone (tRCP), et ce, peu importe l'âge des patients [1]. Cette tRCP permet au témoin d'être guidé et encouragé tout au long de la réalisation des gestes de survie qui peuvent se limiter à la réalisation des compressions thoraciques externes (CTE). La défibrillation doit être la plus précoce possible si le rythme est choquable [2]. En France, les AC sont souvent pris en charge par une équipe du Service mobile d'urgence et de réanimation (Smur), qui se déplace initialement sur le lieu même de l'AC. Dans de nombreux autres pays, notamment anglo-saxons, ce sont des paramedics qui débutent la prise en charge avant de conduire les patients auprès du personnel médical hospitalier. Des critères pronostiques existent, mais ils sont peu utilisés en pratique $[3,4]$. L'âge des patients en $\mathrm{AC}$ pris en charge par les équipes préhospitalières est de plus en plus important. Certaines études ont suggéré que le taux de récupération d'une activité cardiaque spontanée (RACS) est plus important en cas de rythme choquable initial (fibrillation ou tachycardie ventriculaire sans pouls), mais qu'il n'est pas différent selon l'âge des patients réanimés $[5,6]$. D'autres ont permis d'établir des critères péjoratifs de survie tels que l'association « absence de témoin médical de l'AC, rythme cardiaque initial non choquable et absence de RACS après administration de trois doses de $1 \mathrm{mg}$ d'adrénaline [7] ». Ces dernières décennies, l'espérance de vie s'est considérablement accrue et la méde- cine a évolué. En effet, les traitements de l'AC et les gestes de réanimation sont devenus plus agressifs, notamment chez les patients de plus de 65 ans, favorisant ainsi une augmentation des pronostics neurologiques favorables à la suite d'un AC récupéré [8]. Néanmoins, en l'absence de recommandations claires, la durée de réanimation et la décision de non-r éanimation sont laissées à la charge du médecin de l'équipe Smur intervenant sur place.

Le but de ce travail est d'évaluer s'il existe une influence de l'âge du patient sur la durée de réanimation entreprise par les équipes Smur.

\section{Patients et méthodes}

Nous avons réalisé une étude monocentrique ayant analysé rétrospectivement des données collectées de façon prospective. Pour cela, nous avons utilisé les données issues du Registre électronique des arrêts cardiaques (RéAC) qui répertorie prospectivement, depuis juillet 2011, les AC en France. Concernant le Smur de Dijon, 81094 dossiers de régulation médicale (DRM) ont été créés sur l'année 2015, dont 439 pour « arrêt cardiorespiratoire » (ACR), et ce sont 289 Smur qui ont été engagés en missions primaires pour cette étiologie (selon la classification CIM-10 : AC réanimé avec succès, $\mathrm{AC}$ sans précision, décès sans témoin, autres causes de mortalité mal définies et non précisées).

Les données récoltées ont été complétées par celles du DRM, issues du logiciel Centaure $15^{\circledR}$ (Sis France, Courbevoie, France), et celles du dossier patient, issues du logiciel DXCare $^{\circledR}$ (Medasys, Groupe Dedalus, Clamart, France) pour les patients hospitalisés au Centre hospitalier universitaire (CHU) de Dijon. Il faut noter que les véhicules Smur de Dijon sont équipés de GPS (global positioning system) couplé à la régulation du Centre 15. L'ordre de mission arrive directement sur le GPS, automatiquement configuré, facilitant ainsi la recherche de l'adresse du lieu d'intervention.

Les données dijonnaises ont été comparées aux données nationales retrouvées en libre accès sur le site RéAC de l'université Lille-II (http://registreac.org/).

L'ensemble des AC survenus entre le $1^{\mathrm{er}}$ janvier 2015 et le 31 décembre 2015 pour lesquels une équipe Smur du CHU de Dijon a été engagée ont été inclus. Les lieux d'intervention et leur distance de Dijon ainsi que les horaires d'appel, de décision d'envoi d'une équipe Smur, de départ de cette équipe et d'arrivée sur les lieux d'intervention ont été répertoriés. Ces paramètres ont permis de calculer les distances d'intervention (Dijon-lieu d'intervention) grâce à Google Maps ${ }^{\circledR}$ (Google, CA, États-Unis), les délais de prise de décision (heure d'appel-heure de décision d'envoi d'une équipe Smur) et les délais d'arrivée de l'équipe Smur (heure de départ-heure d'arrivée). Nous avons également relevé les durées de réanimation, qu'elles aboutissent à un décès ou à 
une RACS ; la RACS étant définie par un pouls perçu durant une minute minimum. L'évolution neurologique des patients réanimés a également été étudiée grâce à la classification Cerebral Performance Category (CPC) 30 jours après l'AC.

Ont été exclus de l'étude les patients découverts en état de rigidité cadavérique, ceux ayant exprimé ou rédigé des directives anticipées de non-réanimation et les patients n'ayant bénéficié d'aucune réanimation que ce soit par les témoins, les secouristes ou les sapeurs-pompiers.

\section{Analyse statistique}

Les variables qualitatives ont été exprimées en termes d'effectif et de pourcentage et comparées par le test du $\mathrm{Chi}^{2}$. Les variables quantitatives ont été exprimées en termes de différence moyenne avec un intervalle de confiance à $95 \%$ (IC $95 \%$ ) et analysées par le test non paramétrique de Mann-Whitney. Toutes les analyses ont été effectuées avec un risque de première espèce alpha égal à 0,05 . Le tableau Excel regroupant toutes les informations nécessaires à cette étude n'a été utilisé qu'après anonymisation des données afin de respecter la confidentialité des informations relatives à la santé ( $\mu$-argus, institut CBS, Utrecht, Pays-Bas).

\section{Résultats}

En 2015, 253 AC pris en charge par le Smur de Dijon et pour lesquels une réanimation a été proposée ont été recensés. Parmi ceux-ci, 32 patients n'ont bénéficié d'aucune réanimation que ce soit par les témoins, les secouristes ou les sapeurs-pompiers (Fig. 1). Sur les 221 patients en AC étudiés, $130(59 \%)$ avaient un âge inférieur à 75 ans. La population masculine est majoritaire $(72 \%$ pour les moins de 75 ans et $54 \%$ chez les plus de 75 ans). Concernant l'appel au secours sur le territoire dépendant du Smur de Dijon, le numéro composé en première intention est le $18(61 \%)$ pour $37 \%$ d'appels directs au 15. Cependant, il existe une différence significative à Dijon pour l'appel au 15, puisque la moitié des appelants pour les plus de 75 ans le composent en première intention contre moins de $29 \%$ pour les moins de 75 ans (Tableau 1). L'alerte est plus donnée par les professionnels de santé pour les patients de plus de 75 ans, à $30 \%$, alors qu'ils ne sont que $9 \%$ pour les moins de 75 ans $(p<0,001$, Tableau 1).

Le délai entre l'heure d'appel et l'heure de décision d'envoi d'un Smur est en moyenne de huit minutes pour les patients de moins de 75 ans vs neuf minutes pour les patients âgés de 75 ans et plus (différence moyenne $=-2,5$ minutes ; IC $95 \%:[-6 ; 1] ; p=0,13)$. Une différence significative est retrouvée au niveau du délai d'arrivée du Smur. Ainsi, l'équipe de réanimation met en moyenne 11 minutes à se rendre auprès des personnes de moins de 75 ans, contre neuf minutes pour les plus de 75 ans $(p=0,02)$. Cette différence ne s'explique pas par la distance entre Dijon et le lieu d'intervention $(p=0,12)$.

La majorité des patients est en asystolie à l'arrivée du Smur quel que soit l'âge (78\% pour les moins de 75 ans et $70 \%$ pour les plus de 75 ans). Parmi les 188 victimes d'AC ayant bénéficié d'une RCP par l'équipe Smur, le taux de RACS, qui est de $39 \%$, ne dépend pas de l'âge (Tableau 2). De même, le rythme cardiaque retrouvé à la prise en charge n'a pas d'influence significative sur les taux de RACS ou de décès entre les plus et les moins de 75 ans (Tableau 2). Il n'a pas non plus d'influence sur les durées de réanimation entre ces deux populations d'âge.

Afin d'estimer les durées de réanimation, la population a été séparée en deux sous-groupes : réanimation aboutissant à une RACS et réanimation aboutissant à un décès. Les RACS apparaissent en moyenne après $16 \pm 10$ minutes de réanimation chez les patients de moins de 75 ans et après $14 \pm$ 9 minutes chez ceux de 75 ans et plus. Cette différence (deux minutes ; IC $95 \%$ : $[-2,5 ; 6,5])$ n'est pas significative $(p=0,34)$. En cas d'issue péjorative, la réanimation est plus longue chez les personnes âgées de moins de 75 ans : $29 \pm$ 15 minutes vs $23 \pm 19$ minutes, avec une différence moyenne de six minutes (IC $95 \%$ : $[0,9 ; 11,3] ; p<0,01$ ).

Sur tous les patients pris en charge par l'équipe Smur, seuls $59(31 \%)$ étaient vivants à l'admission à l'hôpital (Tableau 2), 41 patients avaient moins de 75 ans (35\% des moins de 75 ans), 18 étaient âgés de plus de 75 ans (25\% des plus de 75 ans). À 30 jours de l'admission, ce ne sont que 12 (6\%) patients qui sont encore vivants, les données sont manquantes pour un d'entre eux. Parmi ces 12 patients, 8 ont eu un bon pronostic neurologique : CPC 1 et 2 ( 7 de moins de 75 ans et 1 de plus de 75 ans).

\section{Discussion}

Dans notre travail, les résultats retrouvent des durées de réanimation Smur plus longues chez les moins de 75 ans, alors que le délai d'apparition de RACS est identique quel que soit l'âge du patient.

Malgré le faible effectif de cette étude monocentrique, les caractéristiques des patients ainsi que la plupart des résultats obtenus sont comparables aux données nationales accessibles sur la base de données RéAC (http://registreac.org/) : la proportion de femmes est de $36 \%$ (vs $35 \%$ au niveau national), la moyenne d'âge est à 67 ans (vs 65 ans au niveau national), les moins de 75 ans représentaient $59 \%$ des patients en AC (vs $63 \%$ au niveau national).

Sur le territoire du Smur de Dijon, l'appel au « $15 »$ ne représente que $39 \%$ des appels de première intention contre $53 \%$ au niveau national. Une des hypothèses pourrait être 


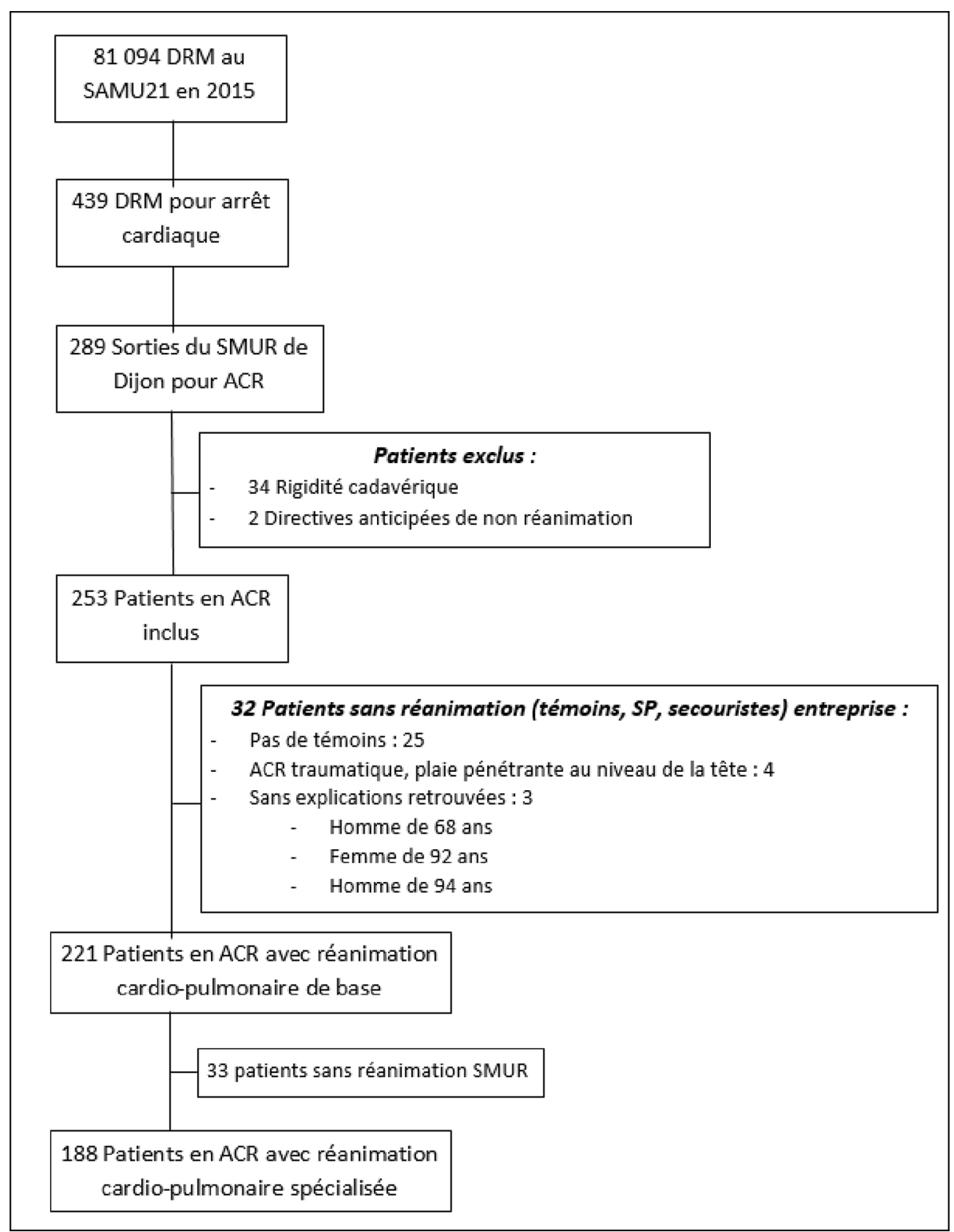

Fig. 1 Organigramme de notre étude concernant les patients victimes d'arrêt cardiaque inclus DRM : dossiers de régulation médicale ; Samu : Service d'aide médicale urgente ; ACR : arrêt cardiorespiratoire, SP : sapeurs-pompiers ; Smur : Service mobile d'urgence et de réanimation

que les habitants des zones rurales sont plus attachés aux secours de proximité, à savoir les sapeurs-pompiers de leur commune, d'où un appel prioritaire au « 18 ». Cependant, dans la population des personnes âgées de plus de 75 ans, le recours au 15 est significativement plus élevé, et ce, surtout par des professionnels de santé. Ces résultats peuvent s'expliquer par le lieu d'habitation plus fréquent en maison de retraite ou en établissement d'hébergement pour personnes âgées dépendantes où ce sont des personnels médicaux et paramédicaux qui sont les témoins des AC et qui préviendraient préférentiellement le Samu grâce à leur sensibilisation et à leur formation aux gestes de premiers secours.

Les délais d'envoi du Smur sont identiques dans les deux groupes. Au niveau du CRRA du Samu 21, des procédures réflexes autorisent les ARM à déclencher conjointement une équipe Smur et un véhicule de secours et d'assistance aux victimes (VSAV), sans régulation préalable par un médecin, lorsqu'ils reconnaissent un AC. L'âge n'est alors pas pris en 


\begin{tabular}{|c|c|c|c|}
\hline & $\begin{array}{l}\text { Patients }< \\
75 \text { ans } \\
n=130\end{array}$ & $\begin{array}{l}\text { Patients } \geq \\
75 \text { ans } \\
n=91\end{array}$ & $\begin{array}{l}\text { Valeur } \\
\text { de } p\end{array}$ \\
\hline \multicolumn{4}{|l|}{ Sexe } \\
\hline Hommes & $93(72)$ & $49(54)$ & 0,20 \\
\hline Femmes & $37(28)$ & $42(46)$ & 0,06 \\
\hline$\hat{A} g e$ & $55 \pm 16$ & $85 \pm 5$ & \\
\hline Hommes & $54 \pm 17$ & $83 \pm 5$ & \\
\hline Femmes & $58 \pm 14$ & $86 \pm 6$ & \\
\hline \multicolumn{4}{|l|}{ Numéro composé } \\
\hline 15 & 37 (29) & $45(49)$ & $<0,05$ \\
\hline 18 & $90(69)$ & 45 (49) & 0,14 \\
\hline $\begin{array}{l}112 \text { et autres } \\
\text { numéros }\end{array}$ & $3(2)$ & $1(1)$ & $>0,99$ \\
\hline \multicolumn{4}{|l|}{ Type d'appelant } \\
\hline Patient & $3(2)$ & $1(1)$ & 0,65 \\
\hline Famille & $74(57)$ & $46(51)$ & 0,61 \\
\hline $\begin{array}{l}\text { Professionnel } \\
\text { de santé }\end{array}$ & $11(9)$ & $27(30)$ & $<0,001$ \\
\hline $\begin{array}{l}\text { Professionnel } \\
\text { de secours }\end{array}$ & $3(2)$ & $6(7)$ & 0,17 \\
\hline Autres & $36(28)$ & $7(7)$ & $<0,01$ \\
\hline Non renseigné & $3(2)$ & $4(4)$ & 0,45 \\
\hline \multicolumn{4}{|c|}{ Rythme à la prise en charge } \\
\hline $\begin{array}{l}\text { Activité } \\
\text { spontanée }\end{array}$ & $4(3)$ & $4(4)$ & 0,72 \\
\hline Asystolie & $104(80)$ & $69(76)$ & 0,80 \\
\hline $\begin{array}{l}\text { Rythme sans } \\
\text { pouls }\end{array}$ & $9(7)$ & $11(12)$ & 0,23 \\
\hline $\mathrm{TV} / \mathrm{FV}$ & $13(10)$ & $7(8)$ & 0,59 \\
\hline
\end{tabular}

TV/FV : tachycardie ventriculaire/fibrillation ventriculaire Les données sont exprimées en nombre (\%). Les âges sont exprimés en moyenne \pm écart-type

compte dans la décision de déclenchement ou non d'une équipe ni dans la régulation préalable par un médecin. Les délais d'intervention des équipes Smur sont quant à eux différents, avec une arrivée sur les lieux d'intervention plus rapide auprès des patients de plus de 75 ans, alors que les distances moyennes de trajet sont similaires. Comme cité précédemment, il existe une proportion plus importante, chez les personnes de plus de 75 ans, de résidents de maison de retraite, dont les adresses sont probablement connues des ambulanciers Smur et rentrées dans le GPS, avec un temps de recherche de l'adresse exacte plus court.

Dans notre étude, le taux de RACS ne dépend pas de l'âge des patients, mais plutôt du rythme initial lors de la prise en charge par l'équipe Smur. La majorité des patients a présenté un rythme cardiaque non choquable à la prise en charge Smur tout âge confondu. Le taux de RACS est très faible
Tableau 2 Descriptif des rythmes cardiaques selon l'issue de la réanimation

\begin{tabular}{|c|c|c|c|}
\hline & $\begin{array}{l}\text { Patients } \\
<75 \text { ans } \\
n=117(62)\end{array}$ & $\begin{array}{l}\text { Patients } \\
\geq 75 \text { ans } \\
n=71(38)\end{array}$ & Valeur de $p$ \\
\hline \multicolumn{4}{|c|}{$\begin{array}{l}\text { Rythme cardiaque à la prise en charge Smur des patients } \\
\text { décédés après réanimation }\end{array}$} \\
\hline Décès & $69(59)$ & $46(65)$ & 0,79 \\
\hline $\begin{array}{l}\text { Activité } \\
\text { spontanée }\end{array}$ & $0(0)$ & $2(3)$ & 0,28 \\
\hline Asystolie & $59(50)$ & $35(49)$ & $>0,99$ \\
\hline $\begin{array}{l}\text { Rythme } \\
\text { électrique } \\
\text { sans pouls }\end{array}$ & $5(4)$ & $5(7)$ & 0,66 \\
\hline $\mathrm{TV} / \mathrm{FV}$ & $5(4)$ & $4(6)$ & 0,96 \\
\hline \multicolumn{4}{|c|}{$\begin{array}{l}\text { Rythme cardiaque à la prise en charge Smur des patients } \\
\text { ayant eu une RACS }\end{array}$} \\
\hline RACS & $48(41)$ & $25(35)$ & $>0,99$ \\
\hline $\begin{array}{l}\text { Activité } \\
\text { spontanée }\end{array}$ & $4(3)$ & $2(3)$ & $>0,99$ \\
\hline Asystolie & $32(27)$ & $15(21)$ & 0,56 \\
\hline $\begin{array}{l}\text { Rythme } \\
\text { électrique } \\
\text { sans pouls }\end{array}$ & $4(3)$ & $5(7)$ & 0,47 \\
\hline $\mathrm{TV} / \mathrm{FV}$ & $8(7)$ & $3(4)$ & 0,70 \\
\hline $\begin{array}{l}\text { Admis vivants } \\
\text { à l'hôpital }\end{array}$ & $41(35)$ & $18(25)$ & 0,39 \\
\hline Vivants à j30 & $9(8)$ & $3(4)$ & 0,56 \\
\hline \multicolumn{4}{|c|}{$\begin{array}{l}\text { Smur : Service mobile d'urgence et de réanimation; TV/FV : } \\
\text { tachycardie ventriculaire/fibrillation ventriculaire ; RACS : } \\
\text { récupération d'une activité cardiaque spontanée } \\
\text { Les données sont exprimées en nombre }(\%)\end{array}$} \\
\hline
\end{tabular}

dans ce cas, sans différence significative selon l'âge des patients. La survie des personnes âgées sur un rythme choquable n'est pas différente de celle des patients plus jeunes, et le délai d'apparition de RACS ne dépend pas de l'âge, alors que l'on réanime significativement moins longtemps les patients de plus de 75 ans [4]. Plusieurs suppositions peuvent expliquer cette réanimation plus courte : la réalisation d'une « réanimation d'attente » qui permet au médecin de recueillir des informations sur la qualité de vie du patient avant un arrêt précoce de soins, une meilleure acceptation du décès lorsque la personne est âgée ou même les sentiments du médecin et de l'équipe refusant de donner des soins leur paraissant disproportionnés par rapport à l'âge avancé des patients. À l'inverse, lorsque la réanimation est un échec, on note qu'elle a été plus longue pour les patients de moins de 75 ans, alors que les RACS apparaissent dans le premier quart d'heure de la réanimation. Dans ce cas, est-il judicieux de poursuivre la réanimation au-delà ? Là aussi, les 
sentiments de la famille ainsi que ceux de l'équipe soignante interviennent probablement. Il est plus difficile d'accepter un décès d'une personne jeune.

Plusieurs études rapportent que le taux de RACS n'est pas lié à l'âge des patients $[3,4]$ et que la survie des personnes âgées sur un rythme choquable n'est pas différente de celle des plus jeunes [9]. Pour d'autres études, le taux de survie est plus important chez les patients de plus de 80 ans avec un rythme choquable que chez les patients plus jeunes avec un rythme non choquable [10]. Une étude taïwanaise a montré que, lorsque l'on ajuste les résultats sur le sexe et les comorbidités, le taux de survie des patients admis à l'hôpital avec des manœuvres de réanimation n'est pas lié à l'âge [11]. Un autre travail a montré qu'un rythme choquable à la prise en charge et une réanimation réalisée par les témoins avant l'arrivée des secours augmentent le pourcentage de survie, alors que l'âge avancé et l'AC à domicile sont des facteurs diminuant la survie [12]. Face au faible effectif de notre étude, il ne nous est pas possible de tirer ces mêmes conclusions. Dans d'autres études, plus la prise en charge de l'AC des patients de plus de 65 ans est agressive, plus on augmente le nombre de patients avec une évolution neurologique favorable [8]. Toutefois, certains auteurs ont montré que la mortalité à j30 augmente avec l'âge [12], mais que lorsqu'ils survivent à l'hospitalisation, le pronostic neurologique des patients âgés est bon [13].

Notre étude pose la question des ressources tant humaines que matérielles à mettre en œuvre pour la réanimation de personnes âgées en AC. Notre travail ne répond évidemment pas à cette question sociétale, éthique et scientifique. Notre médecine et notre système de santé donnent l'impression de pouvoir prolonger la vie inexorablement, donnant aux patients et aux familles un sentiment d'immortalité. Malgré la loi Leonetti sur la fin de vie, peu de personnes ont désigné une personne de confiance ou émis des directives anticipées. Seulement deux patients ont été exclus de l'étude, car ils avaient bien rédigé des directives anticipées. Les équipes Smur intervenant lors d'un AC sont au cœur de cette problématique, mais idéalement, la décision de non-réanimation ne devrait pas être portée par l'équipe seule. Il conviendrait alors de rechercher les comorbidités du patient, son éventuelle prise en charge par un réseau d'hospitalisation à domicile, de soins palliatifs... ainsi qu'une discussion avec la famille ou les proches présents. Malheureusement, le facteur émotionnel, aigu et brutal (avec nécessité d'une prise de décision dans l'immédiateté de la situation) rend peu propice cette réflexion qui devrait être anticipée.

Compte tenu de notre méthodologie, notre travail présente les limites de toute étude monocentrique avec un effectif modeste, rendant parfois les comparaisons inadaptées. Nous n'avons pas de données à plus long terme pour nos patients, mais des études américaines ont suivi une population de patients de plus de 65 ans ayant présenté un AC. Comme attendu, le taux de survie à un an diminue progressivement avec l'âge, avec des causes légèrement différentes de la population plus jeune $[14,15]$. Nous n'avons pas associé à notre étude un questionnaire aux professionnels de santé qui interviennent dans ces décisions (premiers secours, dont les sapeurs-pompiers, équipe Smur : médecins, infirmiers, ambulanciers mais également médecins régulateurs ou ARM). Ceux-ci nous auraient permis de mettre en lumière certains facteurs pris en compte, peut-être de manière inconsciente, dans leurs décisions d'arrêt des réanimations.

\section{Conclusion}

Notre travail suggère qu'il existe une différence dans la durée de réanimation d'un $\mathrm{AC}$ selon l'âge des patients. Malgré les progrès dans la prise en charge des $\mathrm{AC}$ extrahospitaliers, le taux de survie avec un pronostic neurologique acceptable est faible, ce d'autant que les patients sont âgés. Pourtant, l'âge ne devrait pas être le seul critère qui fasse renoncer à une RCP avancée.

Conflit d'intérêts : les auteurs déclarent ne pas avoir de liens d'intérêts.

\section{Références}

1. Nolan JP, Soar J, Zideman DA, et al (2010) European resuscitation council guidelines for resuscitation 2010. Section 1. Resuscitation 10:1219-76

2. Summary of the main changes in the Resuscitation guidelines ERC GUIDELINES $2015 \mathrm{https} / / /$ cprguidelines.eu/sites/573c777 f5e61585a053d7ba5/assets/573c77d75e61585a083d7ba8/ERC summary_booklet_HRES.pdf (Dernier accès le 31 mai 2018)

3. Morrison L, Visentin L, Kiss A, et al (2006) Validation of a rule for terminaison of resuscitation in out-of-hospital cardiac arrest. N Engl J Med 355:47887

4. Libungan B, Lindgvist J, Strömsöe A, et al (2015) Outof-hospital cardiac arrest in the elderly: a large-scale populationbased study. Resuscitation 94:2832

5. Chien DK, Chang WH, Tsai SH, et al (2008) Outcome of nontraumatic out-of-hospital cardiac arrest in the elderly. Int J Gerontol 2:60-6

6. Hagiwara S, Kaneko M, Murata M, et al (2015) Study on the effectiveness of cardiopulmonary resuscitation in elderly patients presenting with cardiopulmonary arrest on arrival. Intern Med 15:1859-63

7. Jabre P, Bougouin W, Dumas F, et al (2016) Early identification of patients with out-of-hospital cardiac arrest with no chance of survival and consideration for organ donation. Ann Intern Med 11:770-8

8. Inokuchi S, Masui K, Miura K, et al (2015) Changes in treatments and outcomes among elderly patients with out-of-hospital cardiac arrest between 2002 and 2012: a post hoc analysis of the SOS-KANTO 2002 and 2012. Resuscitation 97:76-82

9. Kim C, Becker L, Eisenberg MS (2000) Out-of-hospital cardiac arrest in octogenarians and nonagenarians. Arch Intern Med $22: 3439-43$ 
10. Bonnin M, Pepe P, Clark PJ (1993) Survival in the elderly after out-of-hospital cardiac arrest. Crit Care Med 11:1645-51

11. Harnod D, Huei-Ming Ma M, Chang WH, et al (2013) Mortality factors in out-of-hospital cardiac arrest patients: a nationwide population-based study in Taiwan. Int $\mathrm{J}$ Gerontol 4: 216-20

12. Tanner R, Masterson S, Jensen M, et al (2017) Out-of-hospital cardiac arrests in the older population in Ireland. Emerg Med $\mathrm{J}$ 34:659-64
13. Beesems S, Blom M, Van der Pas M, et al (2015) Comorbidity and favorable neurologic outcome after out-of-hospital cardiac arrest in patients of 70 years and older. Resuscitation 94:33-9

14. Chan P, Nallamothu B, Krumholz H, et al (2013) Long-term outcomes in elderly survivors of in-hospital cardiac arrest. N Engl $\mathrm{J}$ Med 11:1019-26

15. Tresch DD, Thakur R, Hoffmann RG, et al (1989) Should the elderly be resuscitated following out-of-hospital cardiac arrest? Am J Med 2:145-50 